\title{
Phase transitions for scaling of structural correlations in directed networks
}

\author{
Pim van der Hoorn and Nelly Litvak \\ Department of Electrical Engineering, Mathematics and Computer Science, University of Twente, Enschede, Netherlands
}

(Received 13 April 2015; published 7 August 2015)

\begin{abstract}
Analysis of degree-degree dependencies in complex networks, and their impact on processes on networks requires null models, i.e., models that generate uncorrelated scale-free networks. Most models to date, however, show structural negative dependencies, caused by finite size effects. We analyze the behavior of these structural negative degree-degree dependencies, using rank based correlation measures, in the directed erased configuration model. We obtain expressions for the scaling as a function of the exponents of the distributions. Moreover, we show that this scaling undergoes a phase transition, where one region exhibits scaling related to the natural cutoff of the network while another region has scaling similar to the structural cutoff for uncorrelated networks. By establishing the speed of convergence of these structural dependencies we are able to assess statistical significance of degree-degree dependencies on finite complex networks when compared to networks generated by the directed erased configuration model.
\end{abstract}

DOI: 10.1103/PhysRevE.92.022803

PACS number(s): 64.60.aq, 02.50.-r

\section{INTRODUCTION}

The tendency of nodes in a network to be connected to nodes of similar large or small degree, called network assortativity, degree mixing, or degree-degree dependency, is an important characterization of the topology of the network, influencing many processes on the network. It has received significant attention in the literature, for instance, in the field of network stability [1], attacks on P2P networks [2], and epidemics [3,4].

An important method to analyze these degree-degree dependencies, or their influence on other network properties or processes on the network, is to compare results to an average over several instances of similar networks with neutral mixing. These null models often come in two flavors. The first approach is to sample from graphs with the same degree sequence but neutral mixing. A widely accepted methodology for such sampling is through the local rewiring model, [5], which takes the original network and randomly swaps edges until a randomized version is attained. The disadvantage of these methods is that they have no theoretical performance guarantees. The second approach is to generate a random graph with neutral mixing, which preserves basic features, such as the degree distribution. A well known model of this type is the configuration model (CM) [6-8]. Here the degrees of vertices are drawn independently from the given distribution, under the restriction that the total sum of degrees is even. Then the stubs are paired uniformly at random to form edges. If we want to obtain a simple graph in this way, we can either rewire till a simple graph is generated (repeated configuration model), or we remove the excess edges and self loops (erased configuration model).

We note that there are many other methods that generate simple random graphs and have theoretically established performance guarantees. For example, sequential algorithms based on the properties of graphical sequences were proposed for undirected networks $[9,10]$ and directed networks [11]. Another example is a grand-canonical model in [12] that generates a graph with given average degrees using a maximum-entropy method. However, to the best of our knowledge, none of these methods has an efficient implementation. Even the complexity $O(N E)$ in $[10,11]$, where $N$ is the network size and $E$ the number of edges, is arguably not feasible for truly large networks, such as Wikipedia or Twitter.

Although for both local rewiring and the configuration model neutral mixing is expected, since there is no preference in connecting two vertices, negative correlations are observed [13-15] for scale-free networks with infinite variance of degrees, i.e., where the degree distribution satisfies

$$
P(k) \sim k^{-(\gamma+1)}, \quad 1<\gamma \leqslant 2 .
$$

In [14] this phenomenon is explained by observing that if one allows at most one edge between two vertices, nodes with large degree must connect to nodes of small degree because there are simply not enough distinct large nodes to connect to. A similar explanation is given in [13]. Here, however, this is then related to the difference in scaling between the natural and structural cutoff of the network. The former is defined [16] as the degree value $k_{c}$, of which, on average, only one instance is observed:

$$
N \int_{k_{c}}^{\infty} P(k) d k \sim 1 .
$$

The structural cutoff is defined as the value $k_{s}$ for which the ratio between the average number of edges that connect any two vertices of degree $k_{s}$, and the maximum possible number of such edges in a simple graph, is 1 . For networks with degree distribution (1) it follows from (2) that the natural cutoff scales as $N^{1 / \gamma}$, while the structural cutoff for uncorrelated networks scales as $N^{1 / 2}$; see [17]. Therefore, when $\gamma<2$, the natural cutoff scales at a slower rate which in turn gives rise to structural negative correlations.

To remedy these finite size effects the authors of [13] propose an uncorrelated configuration model. This model follows the same procedure as the regular configuration model, with the addition that the sampled degrees are bounded, $m \leqslant k_{i} \leqslant$ $N^{1 / 2}$. Experiments in [13] indeed show that these networks are uncorrelated. However, many scale-free networks, for instance Twitter, have nodes whose degree is of larger order than $N^{1 / 2}$, which is a characteristic property of scale-free graphs. For example, Table I displays the characteristics of Wikipedia networks for different languages. Here we see that the maximum out-degree could be considered to be of 
TABLE I. Basic degree characteristics of Wikipedia networks. The exponents of the degree distributions are estimated using the implementation of the techniques from [18] by Bloem [19].

\begin{tabular}{lrrrrrr}
\hline \hline Wikipedia & $N$ & $N^{1 / 2}$ & $\gamma_{+}$ & $\gamma_{-}$ & $\max D^{+}$ & $\max D^{-}$ \\
\hline DE & 1532978 & 1238 & 1.80 & 1.05 & 5032 & 118064 \\
EN & 4212493 & 2052 & 2.14 & 1.20 & 8104 & 432629 \\
IT & 1017953 & 1009 & 1.96 & 1.05 & 5212 & 91588 \\
NL & 1144615 & 1070 & 1.82 & 1.10 & 10175 & 102450 \\
PL & 949153 & 974 & 1.90 & 1.04 & 4100 & 112537 \\
\hline \hline
\end{tabular}

order $N^{1 / 2}$, while the maximum in-degree is definitely of a much larger scale. Therefore, randomized versions of these networks, generated by the uncorrelated configuration model, do not have the same basic degree characteristics as the original network, since the maximum degree is restricted. Hence, they are less suitable for comparison of the degree-degree dependencies.

In this paper we consider the directed erased configuration model (ECM) [20], where, after the pairing, self-loops are removed and multiple edges are merged. In our recent work [21], Sec. 5, we showed that this model has neutral mixing in the infinite network size limit. The idea behind this result is that the total average number of erased edges per node, which defines the difference in the correlations between the $\mathrm{CM}$ and the ECM, goes to zero when the size of the network grows. By this result, from a purely mathematical point of view the ECM is a null model for degree-degree dependencies in the limit. Moreover, asymptotically, the degree distributions and hence all basic degree characteristics are preserved. Still, for finite sizes, structural dependencies are present.

Rather than trying to control these correlations, our goal is to evaluate their magnitude and investigate their size dependence. We obtain the scaling for the structural correlations in the ECM, in terms of the power law exponents of the in- and out-degrees. In particular, we show that this scaling undergoes an interesting phase transition, and can be dominated by terms related to either the structural or the natural cutoff of the network. To the best of our knowledge, this is the first study that provides a systematic mathematical characterization for the magnitude of negative correlations in a simple graph with neutral mixing.

By determining the scaling of the structural correlations we can assess the significance of measured correlations as well as their influence on network processes, on real world networks of finite size, by comparing them to the directed erased configuration model. This approach has the advantage of preserving the degree characteristics of the original network; it can be easily implemented and applied to all networks with scale-free degree distributions and finite expectation.

\section{DEGREE-DEGREE DEPENDENCIES IN RANDOM DIRECTED NETWORKS}

We analyze degree-degree dependencies in random directed networks of size $N$, where the distribution of the out- and in-degrees $\left(D^{+}, D^{-}\right)$follows, respectively,

$$
P^{+}(k) \sim k^{-\left(\gamma_{+}+1\right)} \text { and } P^{-}(\ell) \sim \ell^{-\left(\gamma_{-}+1\right)}, \gamma_{ \pm}>1 \text {. }
$$

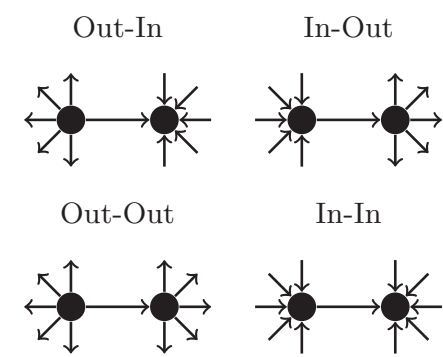

FIG. 1. The four different degree-degree dependency types in directed networks.

In directed networks one can consider four types of degreedegree dependencies, depending on the choice of the degree type on both sides of an edge; see Fig. 1. For the remainder of this paper we denote by $E$ the number of edges and adopt the notation style from $[22,23]$ to index the degree types by $\alpha, \beta \in\{+,-\}$.

A common measure for degree-degree dependencies, introduced in [24], computes Pearson's correlation coefficients on the joint data $\left(D_{i}^{\alpha}, D_{j}^{\beta}\right)_{i \rightarrow j}$, where the indices run over all $i, j$ for which there is an edge $i \rightarrow j$.

However, Pearson's correlation coefficients are unable to measure strong negative degree-degree dependencies in large networks where the variance of the degrees is infinite, as was shown for undirected networks in $[25,26]$ and for directed networks in [23]. Since our interest is mainly in networks in the infinite variance domain, i.e., $1<\gamma_{ \pm} \leqslant 2$, we need different measures. In [23] it was suggested to use rank correlations, related to Spearman's rho [27] and Kendall's tau [28], to measure degree-degree dependencies.

Spearman's rho computes Pearson's correlation coefficient on the ranks of $\left(D_{i}^{\alpha}, D_{j}^{\beta}\right)_{i \rightarrow j}$ rather then their actual values. Since this data will contain many ties, one needs to use ranking schemes that deal with these ties. In [23] two such schemes are considered, resolving ties at random and assigning an average rank to tied values, which give two correlation measures denoted by $\rho_{\alpha}^{\beta}$ and $\bar{\rho}_{\alpha}^{\beta}$, respectively. Here, the subscript index denotes the degree type of the source, while the superscript index denotes the degree type of the target of a directed edge. For instance, $\rho_{+}^{-}$denotes Spearman's rho for the out-in dependency. The second rank correlation measure, Kendall's tau $\tau_{\alpha}^{\beta}$, calculates the normalized number of swaps needed to match the ranks of the joint data.

Exact formulas for these three measures, in terms of the degrees, are given in [23]. In [21] formulas are given in terms of the empirical distributions of $D^{\alpha}$ and $D^{\beta}$ and their joint distribution, evaluated at $\left(D_{i}^{\alpha}, D_{j}^{\beta}\right)$ for an edge $i \rightarrow j$ selected uniformly at random. From these it follows that if the network has neutral mixing, then $\rho_{\alpha}^{\beta}$ and $\tau_{\alpha}^{\beta}$ are similar, while $\rho_{\alpha}^{\beta}$ and $\bar{\rho}_{\alpha}^{\beta}$ differ by a term of $O(1)$, which does not influence the scaling. To illustrate this we plotted the empirical cdf's of $\rho_{+}^{-}$, $\bar{\rho}_{+}^{-}$, and $\tau_{+}^{-}$for a collection of ECM graphs in Fig. 2, where we clearly observe the similar behavior of the three measures. Therefore, for the analysis of degree-degree dependencies, we will only consider $\rho_{\alpha}^{\beta}$, which corresponds to Spearman's rho where ties are resolved uniformly at random. 


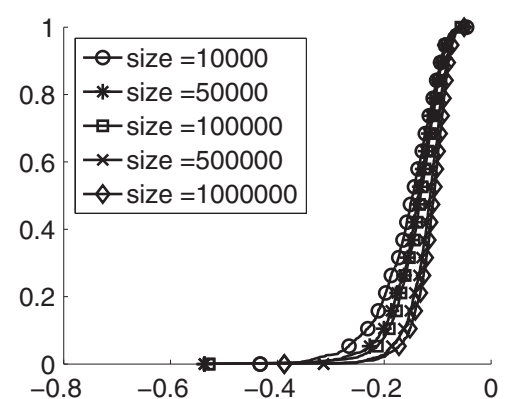

(a) $\rho_{+}^{-}$

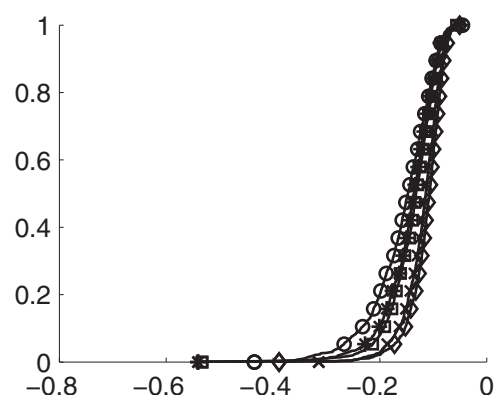

(b) $\bar{\rho}_{+}^{-}$

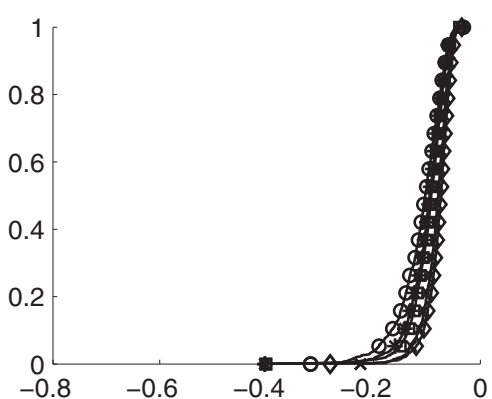

(c) $\tau_{+}^{-}$

FIG. 2. Plots of the empirical cumulative distribution of $\rho_{+}^{-}, \bar{\rho}_{+}^{-}$, and $\tau_{+}^{-}$for ECM graphs of different sizes with $\gamma_{ \pm}=1.2$. Each plot is based on $10^{3}$ realizations of the model.

\section{THE DIRECTED ERASED CONFIGURATION MODEL}

The directed configuration model (CM) starts with a degree sequence $\left(D_{i}^{+}, D_{i}^{-}\right)_{1 \leqslant i \leqslant N}$ that satisfies, for some $\mu>0$,

$$
\begin{aligned}
& E=\sum_{i=1}^{N} D_{i}^{ \pm} \sim \mu N \\
& \sum_{i=1}^{N} D_{i}^{+} D_{i}^{-} \sim \mu^{2} N \\
& \sum_{i=1}^{N}\left(D_{i}^{ \pm}\right)^{p} \sim N^{p / \gamma_{ \pm}}, \quad p>\gamma_{ \pm} .
\end{aligned}
$$

The stubs are then paired at random to form edges. This will in general constitute a graph with self-loops and multiple edges between nodes. If the degree variance is finite, then the probability of generating a simple graph is bounded away from zero and thus, by repeating the pairing step until such a graph is generated, we get a network randomly sampled from all networks of given size and degree sequences. This is called the repeated configuration model (RCM).

When the variance of the degrees is infinite, the probability of generating a simple graph converges to zero as the graph size increases, and therefore we need to enforce that the resulting graph is simple. For this we use the erased configuration model (ECM), where, during the pairing, a new edge is removed if it already exists or if it is a self-loop. Although this seems to be a strong alteration of the initial degree sequence, asymptotically, the degrees of the resulting network still follow the same distribution; see [20]. For illustration, in Fig. 3 we plotted the degree distributions of and ECM graph of size $10^{6}$ before and after the removing of edges. Clearly there is hardly any difference between the two distributions. In particular the degree sequences of ECM graphs still satisfy (4). Unlike many other methods, random pairing of the stubs can be implemented very efficiently for even billions of nodes. Moreover, the ECM is computationally less expensive than the RCM, since we do not need to repeat the pairing. Therefore we suggest to use the ECM as a standard null model. In the rest of the paper we will characterize the structural dependencies in the ECM.

\section{DEGREE-DEGREE DEPENDENCIES IN THE ECM}

It is clear that when we use the CM, i.e., allow for multiple edges and self-loops, then our graphs will have neutral mixing since all stubs are connected completely at random. For the ECM, however, we remove edges to make the graph simple, which has been shown $[13,14]$ to give rise to negative correlations. Nevertheless, the ECM has asymptotically neutral mixing, which can be shown as follows.

Let $E_{i j}$ be the matrix counting the number of edges between $i$ and $j$ after the pairing and let $E_{i j}^{c}$ denote the matrix counting the number of removed edges between $i$ and $j$ by the ECM. Then for the CM it holds that $D_{i}^{+}=\sum_{j=1}^{N} E_{i j}$ while for the ECM we have $D_{i}^{+\prime}=\sum_{j=1}^{N}\left(E_{i j}-E_{i j}^{c}\right)$. Therefore, the difference between the empirical distributions of $D_{i}^{\alpha}$ and $D_{j}^{\beta}$, for an edge $i \rightarrow j$ sampled at random, in the CM and ECM, will be of the order $\sum_{i, j=1}^{N} E_{i j}^{c} / E$, whose average, with respect to the degree sequences, converges to zero [21]:

$$
\lim _{N \rightarrow \infty} \frac{1}{N} \sum_{i, j=1}^{N}\left\langle E_{i j}^{c}\right\rangle=0 .
$$

This implies that the values of $\rho_{\alpha}^{\beta}$ for an ECM graph will converge to that of a CM graph, hence, asymptotically, $\rho_{\alpha}^{\beta}=0$ and also $\bar{\rho}_{\alpha}^{\beta}=0=\tau_{\alpha}^{\beta}$, for the ECM.

However, for finite realizations in the infinite variance regime, negative correlations are still observed. To illustrate this we plotted the empirical cumulative distribution functions of $\rho_{\alpha}^{\beta}$ for graphs generated by the ECM with both finite and infinite degree variance; see Figs. 4 and 5, respectively. In addition, Table II contains the average values for all four correlation types in the infinite variance regime. One immediately observes that the out-in dependency in ECM graphs with infinite variance, Fig. 5(a), displays strong structural negative correlations which decrease as the network grows, while for the other three dependency types the values are concentrated around zero. Moreover, we see in Fig. 4 that all four dependency types behave similarly when the variance of the degrees is finite.

These negative out-in correlations $\left(\rho_{+}^{-}\right)$can be explained by first observing that multiple edges are more likely to start in a node of large out-degree and end in a node of large in-degree, since these are more likely to be sampled. 


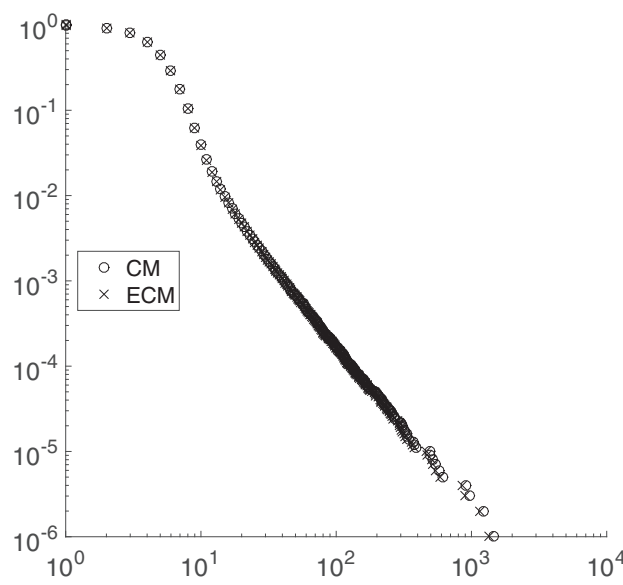

(a) Out-degrees

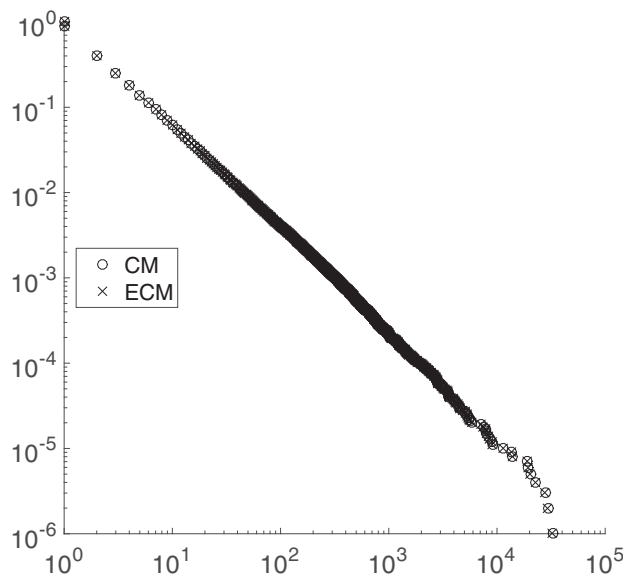

(b) In-degrees

FIG. 3. Plots of the out- and in-degree distribution, on log-log scale, for a graph generated by the ECM, of size $10^{6}$ with $\gamma_{+}=1.9$ and $\gamma_{-}=1.2$, before $(\mathrm{CM})$ and after (ECM) the removal of edges.

Now, consider the algorithm as first connecting all stubs at random and then removing self-loops and merging multiple edges. By construction, immediately after the pairing the network will have neutral mixing. When merging multiple edges we will often delete connections from nodes of large out-degree to nodes of large in-degree. Such edges have contributed positively into $\rho_{+}^{-}$, thus, deleting them will shift $\rho_{+}^{-}$from zero in the $\mathrm{CM}$ to a negative value in the
ECM. The other three dependency types are not effected since the out- and in-degrees of a node in the ECM are independent.

Motivated by the analysis in this section, we will further focus on the behavior of $\rho_{+}^{-}$in the infinite-variance case, $1<$ $\gamma_{+}, \gamma_{-} \leqslant 2$, as the only scenario where we observe prominent structural correlations. We will discuss other scenarios in Sec. VI.

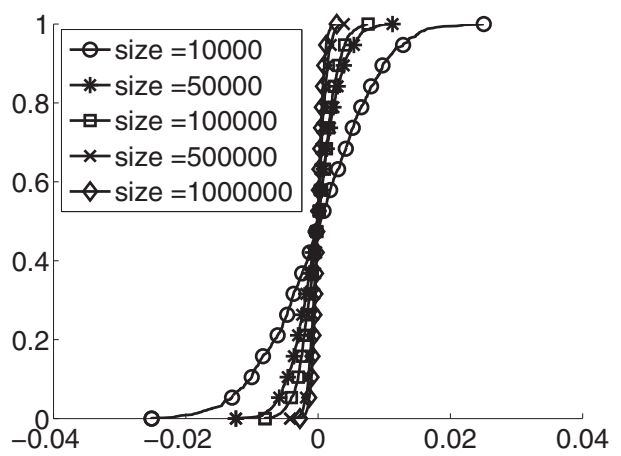

(a) Out-In

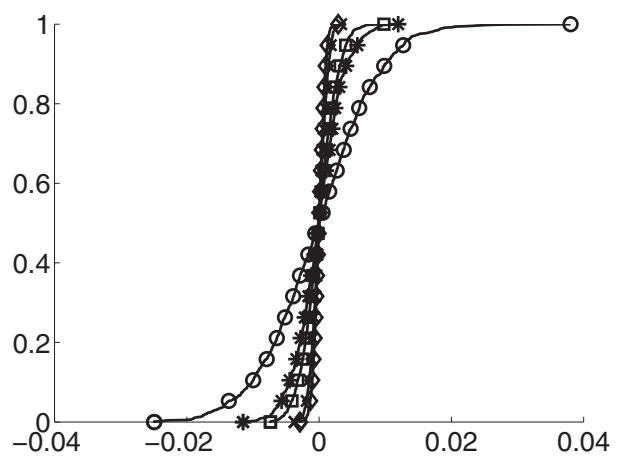

(c) Out-Out

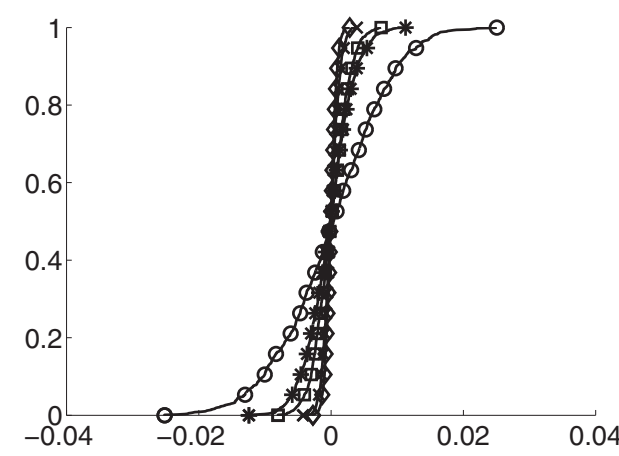

(b) In-Out

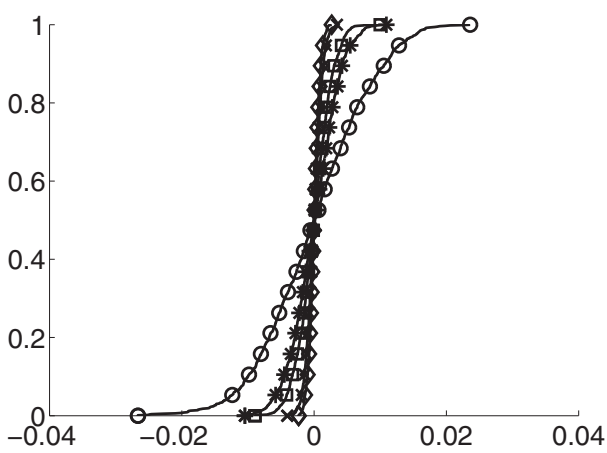

(d) In-In

FIG. 4. Plots of the empirical cumulative distribution of $\rho_{\alpha}^{\beta}$ for all four degree-degree dependency types for ECM graphs of different sizes with $\gamma_{ \pm}=2.1$. Each plot is based on $10^{3}$ realizations of the model. 


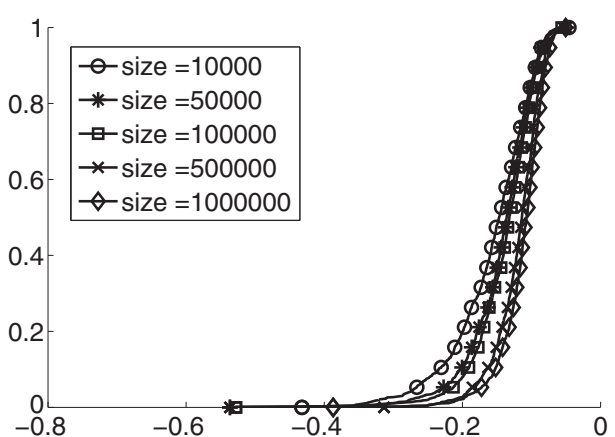

(a) Out-In

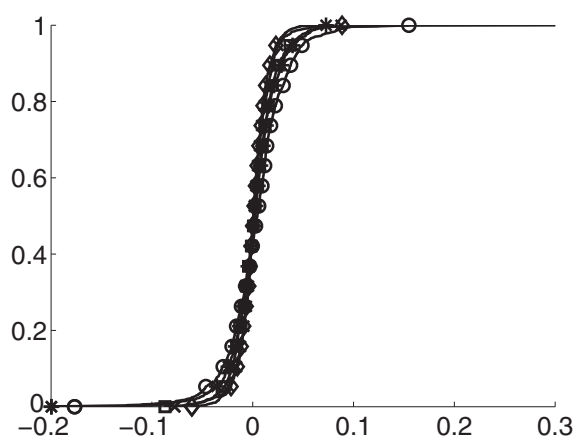

(c) Out-Out

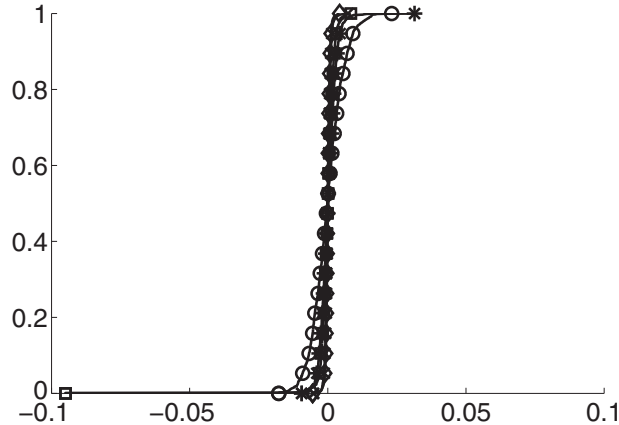

(b) In-Out

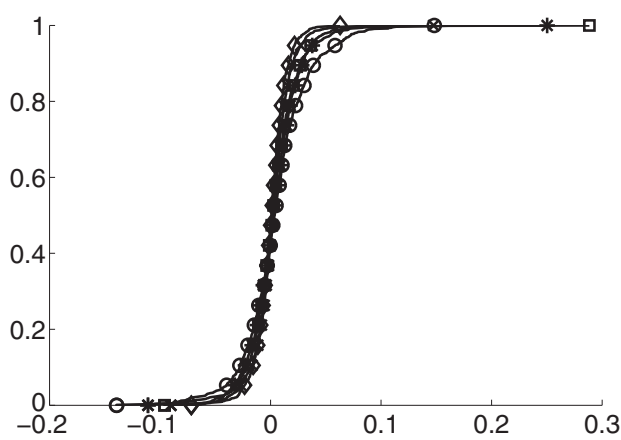

(d) In-In

FIG. 5. Plots of the empirical cumulative distribution of $\rho_{\alpha}^{\beta}$ for all four degree-degree dependency types for ECM graphs of different sizes with $\gamma_{ \pm}=1.2$. Each plot is based on $10^{3}$ realizations of the model.

\section{SCALING OF THE OUT-IN DEGREE-DEGREE DEPENDENCY IN THE ECM}

We will determine the scaling of $\rho_{+}^{-}$as a function of the exponents $\gamma_{ \pm}$. That is, we will find coefficients $f\left(\gamma_{+}, \gamma_{-}\right)$such that

$$
\frac{\rho_{+}^{-}-\left\langle\rho_{+}^{-}\right\rangle}{N^{f\left(\gamma_{+}, \gamma_{-}\right)}}
$$

converges to some limiting distribution. Here the expectation $\left\langle\rho_{+}^{-}\right\rangle$is taken over all possible graphs of size $N$, generated by the ECM, with degree sequences satisfying (4). We note that although $\left\langle\rho_{+}^{-}\right\rangle$is of similar order as the typical spreading of $\rho_{+}^{-}$, the latter, which we are going to evaluate, will define the magnitude of the structural negative correlations.

We obtain the scaling exponents $f\left(\gamma_{+}, \gamma_{-}\right)$by establishing upper bounds on the scaling, and then show empirically that

TABLE II. The average values for $\rho_{\alpha}^{\beta}$ for all four degree-degree dependency types, for ECM graphs of different sizes, with $\gamma_{ \pm}=1.2$, based on $10^{3}$ realizations of the model.

\begin{tabular}{lcrcc}
\hline \hline$N$ & $\left\langle\rho_{+}^{-}\right\rangle$ & \multicolumn{1}{c}{$\left\langle\rho_{-}^{+}\right\rangle$} & $\left\langle\rho_{+}^{+}\right\rangle$ & $\left\langle\rho_{-}^{-}\right\rangle$ \\
\hline 10000 & -0.1568 & -0.0001 & 0.0039 & 0.0048 \\
50000 & -0.1439 & 0.0001 & 0.0014 & 0.0029 \\
100000 & -0.1388 & -0.0001 & 0.0026 & 0.0028 \\
500000 & -0.1198 & 0.0001 & 0.0011 & 0.0017 \\
1000000 & -0.1131 & 0.0000 & 0.0009 & 0.0002 \\
\hline \hline
\end{tabular}

these bounds are tight. The scaling is an important quantity, characterizing the spread around the sample mean of $\rho_{+}^{-}$as a function of $N$. Roughly, this tells us how much the measured values on a ECM graph of size $N$ can deviate from the average and therefore enable us to assess the significance of the measured correlations of the corresponding real world networks.

\section{A. Scaling of the erased number of edges}

As we discussed in the previous section, the structural negative correlations appear after multiple edges and self-loops are erased. Hence, part of the scaling of $\rho_{+}^{-}$comes from the scaling of the average total number of erased edges. The latter scaling has a phase transition, which we will show by establishing two different upper bounds.

For the first upper bound, observe that

$$
\sum_{i, j=1}^{N} E_{i j}^{c}=\sum_{i=1}^{N} S_{i i}+\sum_{i, j=1}^{N} M_{i j}
$$

where $S$ is the diagonal matrix counting the number of self loops and $M$ is the zero diagonal matrix that counts the excess edges, so $M_{i j}=k>0$ means that $E_{i j}=k+1$. For the selfloops it holds that

$$
\left\langle S_{i i}\right\rangle=\frac{D_{i}^{+} D_{i}^{-}}{E} .
$$


If we now take the total number of pairs of edges between $i$ and $j$ as an upper bound for $M_{i j}$, then

$$
\left\langle M_{i j}\right\rangle \leqslant \frac{\left(D_{i}^{+}\right)^{2}\left(D_{j}^{-}\right)^{2}}{E^{2}} .
$$

Applying (7) and (8) to (6) we get

$$
\sum_{i, j=1}^{N} \frac{\left\langle E_{i j}^{c}\right\rangle}{E} \leqslant \frac{\sum_{i, j=1}^{N}\left(D_{i}^{+}\right)^{2}\left(D_{j}^{-}\right)^{2}}{E^{3}}+\frac{\sum_{i=1}^{N} D_{i}^{+} D_{i}^{-}}{E^{2}} .
$$

We remark that if the second moment of both the out- and in-degrees exists, then this upper bound scales as $N^{-1}$. When this is not the case, we get the scaling from (4) as

$$
\frac{1}{E} \sum_{i, j=1}^{N}\left\langle E_{i j}^{c}\right\rangle=O\left(N^{\left(2 / \gamma_{+}\right)+\left(2 / \gamma_{-}\right)-3}\right) .
$$

The upper bound (10) is rather crude in the sense that for certain $1<\gamma_{ \pm} \leqslant 2$, we have $\left(2 / \gamma_{+}\right)+\left(2 / \gamma_{-}\right)>3$ so that the right-hand side of (10) becomes infinite as $N \rightarrow \infty$.

To get a more precise upper bound let $p(n, m, L)$ denote the probability that none of the outbound stubs from a set of size $n$ connect to an inbound stub from a set of size $m$, given that the total number of available stubs is $L$. We will establish a recursive relation for $p\left(D_{i}^{+}, D_{j}^{-}, E\right)$ by adopting the analysis from [29], Sec. 4. Similarly we get, by conditioning on whether we pick an inbound stub of $i$ or not,

$$
p\left(D_{i}^{+}, D_{j}^{-}, E\right) \leqslant\left(1-\frac{D_{j}^{-}}{E}\right) p\left(D_{i}^{+}-1, D_{j}^{-}, E-1\right),
$$

where the upper bound comes from neglecting the event $D_{i}^{+}+$ $D_{j}^{-}>E$, in which case $p\left(D_{i}^{+}, D_{j}^{-}, E\right)=0$. Continuing the recursion yields

$$
p\left(D_{i}^{+}, D_{j}^{-}, E\right) \leqslant \prod_{k=0}^{D_{i}^{+}-1}\left(1-\frac{D_{j}^{-}}{E-k}\right),
$$

and a first order Taylor expansion then gives

$$
p\left(D_{i}^{+}, D_{j}^{-}, E\right) \leqslant e^{-D_{i}^{+} D_{j}^{-} / E} .
$$

Now, recall that $E_{i j}$ denotes the total number of edges between $i$ and $j$ in the CM, before the removal step. Therefore,

$$
\left\langle E_{i j}^{c}\right\rangle=\left\langle E_{i j}\right\rangle-\left[1-p\left(D_{i}^{+}, D_{j}^{-}, E\right)\right] .
$$

Since $E=\sum_{i, j=1}^{N}\left\langle E_{i j}\right\rangle$ it follows that

$$
\frac{1}{E} \sum_{i, j=1}^{N}\left\langle E_{i j}^{c}\right\rangle=1-\frac{N^{2}}{E}+\frac{1}{E} \sum_{i, j=1}^{N} p\left(D_{i}^{+}, D_{j}^{-}, E\right) .
$$

Hence, by plugging (11) into (12) we arrive at the following upper bound for the total average number of erased edges:

$$
\frac{1}{E} \sum_{i, j=1}^{N}\left\langle E_{i j}^{c}\right\rangle \leqslant 1-\frac{N^{2}}{E}+\frac{1}{E} \sum_{i, j=1}^{N} e^{-D_{i}^{+} D_{j}^{-} / E} .
$$

The right hand side of (13) can be slightly rewritten to obtain a more informative expression, which is the product of

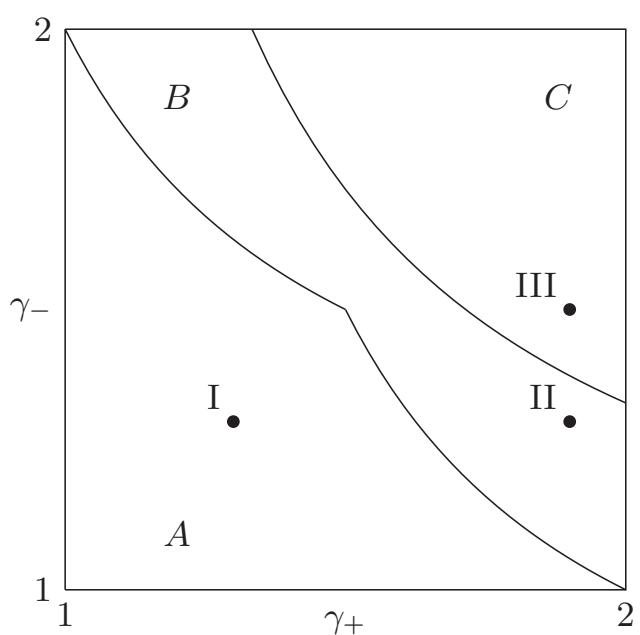

FIG. 6. Plot of the different scaling regimes for $\rho_{+}^{-}$. The scaling terms for each of the three regions can be found in Table III. The roman numerals indicate the three different choices of $\gamma_{+}$and $\gamma_{-}$, used in Figs. 7 and 8, to illustrated the different regimes.

$N^{2} / E$ and the term

$$
\frac{1}{E} \sum_{i, j=1}^{N} \frac{D_{i}^{+} D_{j}^{-}}{N^{2}}-1+\sum_{i, j=1}^{N} \frac{e^{-\left(D_{i}^{+} D_{j}^{-}\right) / E}}{N^{2}} .
$$

Next, we note that (14) can be seen as an empirical form of

$$
\frac{1}{N \mu}\langle\xi\rangle-1+\left\langle e^{-\xi /(N \mu)}\right\rangle,
$$

where, letting $\gamma_{\min }=\min \left\{\gamma_{+}, \gamma_{-}\right\}$, $\xi$ has distribution

$$
P_{\xi}(k) \sim k^{-\left(\gamma_{\min }+1\right)},
$$

and $\langle\xi\rangle=\mu^{2}$. From a classical Tauberian theorem for regularly varying random variables, see for instance [30], Theorem A, it follows that (15) scales as $N^{-\gamma_{\min }}$. When we replace $E$ by $\mu N$ in (14), we obtain

$$
\frac{1}{\mu N} \sum_{i, j=1}^{N} \frac{D_{i}^{+} D_{j}^{-}}{N^{2}}-1+\sum_{i, j=1}^{N} \frac{e^{-D_{i}^{+} D_{j}^{-} /(\mu N)}}{N^{2}}
$$

and observe that (15) is the expectation of (16). The function $f(x)=x-1+e^{-x}$ is positive, hence, it follows that (16) and (15) have the same scaling, $N^{-\gamma_{\min }}$. Finally, the difference between (14) and (16) is dominated by the term

$$
\left|\frac{1}{E}-\frac{1}{\mu N}\right|=O\left(N^{-2}|E-\mu N|\right) .
$$

TABLE III. The three scaling terms for $\rho_{+}^{-}$for each of the three regions displayed in Fig. 6

\begin{tabular}{cc}
\hline \hline Region & $f\left(\gamma_{+}, \gamma_{-}\right)$ \\
\hline A & $1 / \gamma_{\text {min }}-1$ \\
B & $\left(2 / \gamma_{+}\right)+\left(2 / \gamma_{-}\right)-3$ \\
C & $-1 / 2$ \\
\hline \hline
\end{tabular}




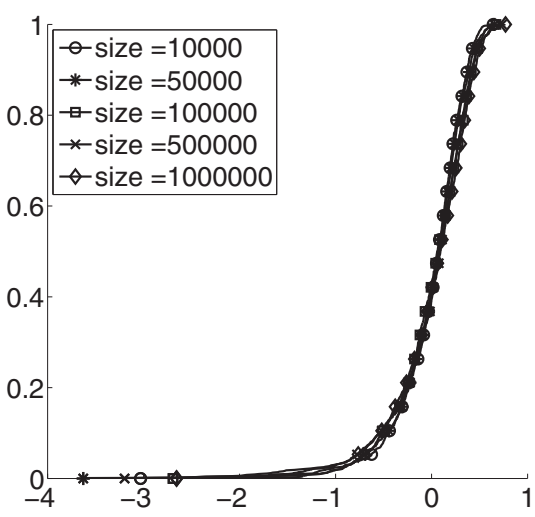

(a) I - $N^{1 / \gamma_{\min }-1}$

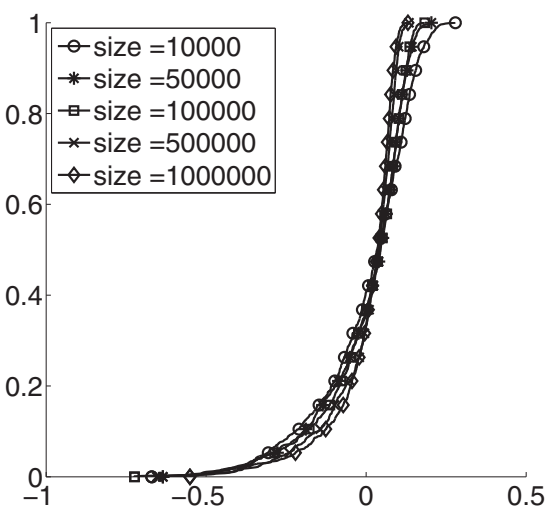

(d) II $-N^{1 / \gamma_{\min }-1}$

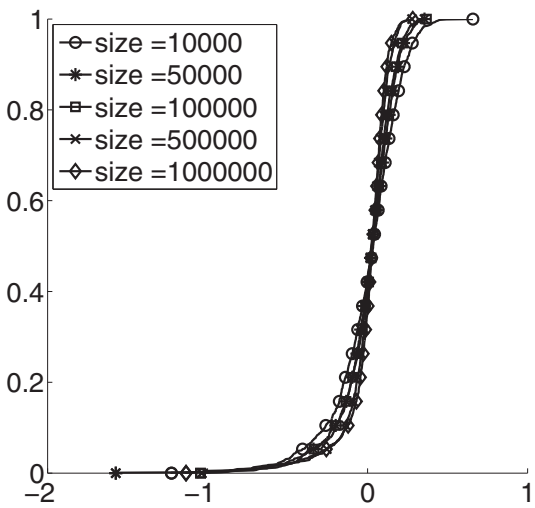

(g) III $-N^{1 / \gamma_{\min }-1}$

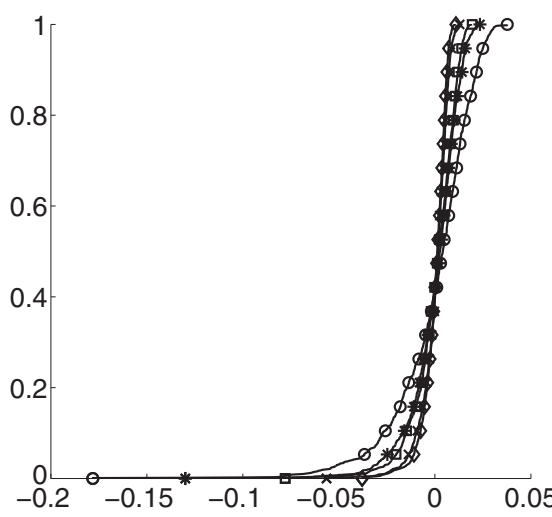

(b) I $-N^{\left(2 / \gamma_{+}\right)+\left(2 / \gamma_{-}\right)-3}$

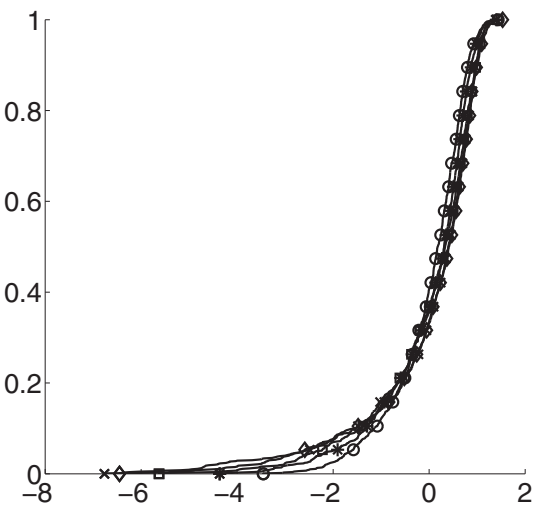

(e) II $-N^{\left(2 / \gamma_{+}\right)+\left(2 / \gamma_{-}\right)-3}$

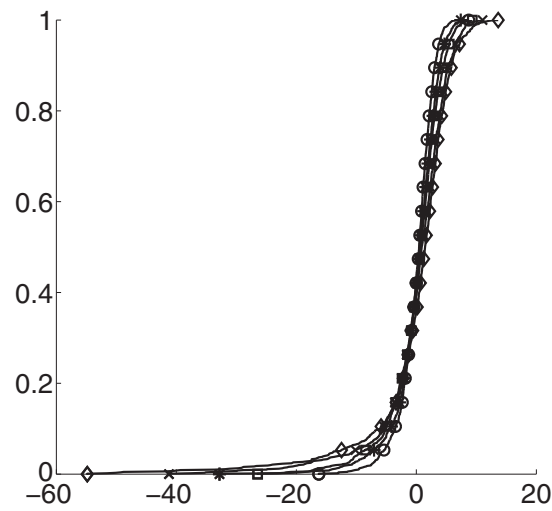

(h) III $-N^{\left(2 / \gamma_{+}\right)+\left(2 / \gamma_{-}\right)-3}$

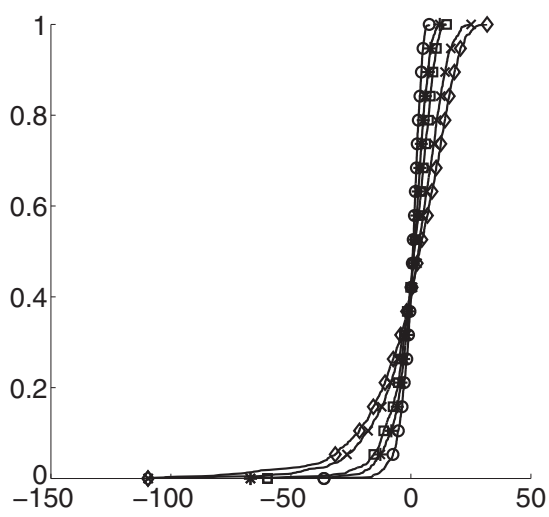

(c) I $-N^{-1 / 2}$

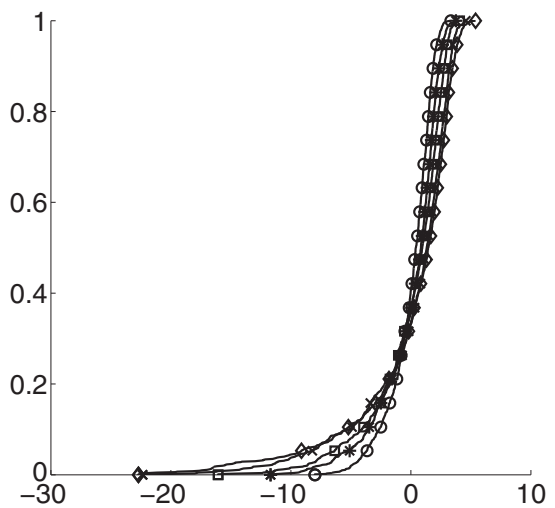

(f) II $-N^{-1 / 2}$

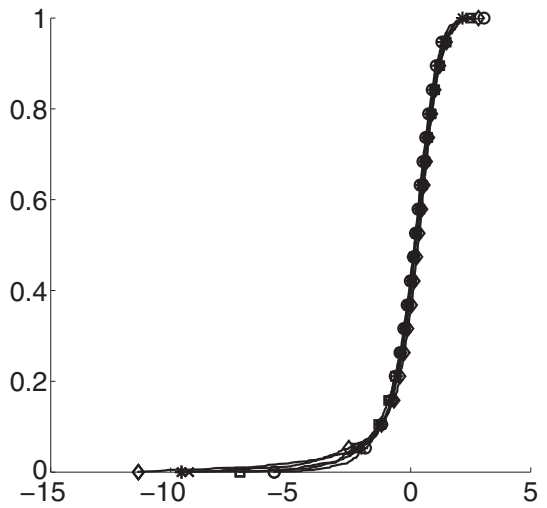

(i) III $-N^{-1 / 2}$

FIG. 7. Plots of the empirical cumulative distribution function of $\rho_{+}^{-}$using different scaling and for different choices of $\gamma_{ \pm}$. The left column is scaled by $N^{1 / \gamma_{\min }-1}$, the center column by $N^{2 / \gamma_{+}+2 / \gamma_{-}-3}$, and the right column by $N^{-1 / 2}$. The first row is for ECM graphs with $\gamma_{ \pm}=1.3$, the second for $\gamma_{+}=1.9, \gamma_{-}=1.3$, and the third for $\gamma_{+}=1.9, \gamma_{-}=1.5$, corresponding to points I, II, and III, respectively, in Fig. 6 .

Recall that $\sum_{i=1}^{n} D_{i}^{+}=E=\sum_{i=1}^{n} D_{i}^{-}$. Hence, we obtain from the central limit theorem for regularly varying random variables, see [31], that

$$
N^{-2}|E-\mu N|=O\left(N^{-2+1 / \gamma_{\min }}\right) .
$$

which dominates $N^{-\gamma_{\min }}$ when $1<\gamma_{ \pm} \leqslant 2$. Summarizing, we have that (14) scales as $O\left(N^{-2+1 / \gamma_{\text {min }}}\right)$ and hence, since $N^{2} / E=O(N)$, it follows that

$$
\frac{1}{E} \sum_{i, j=1}^{N}\left\langle E_{i j}^{c}\right\rangle=O\left(N^{-1+1 / \gamma_{\min }}\right) .
$$

The scaling in (17) is related to that of the structural cutoff described in [17], adjusted to the setting of directed networks with degree distributions (3). Moreover, comparing (17) to (10) we observe a phase transition, with respect to the tail exponents $\gamma_{ \pm}$of the degree distributions, in the scaling of the average total number of removed edges in the ECM, which will induce a phase transition in the scaling of the out-in degree-degree dependency.

\section{B. Phase transitions for the out-in degree-degree dependency}

First we remark that, for the $\mathrm{CM}$, the empirical distribution of the degrees on both sides of a randomly sampled edge 


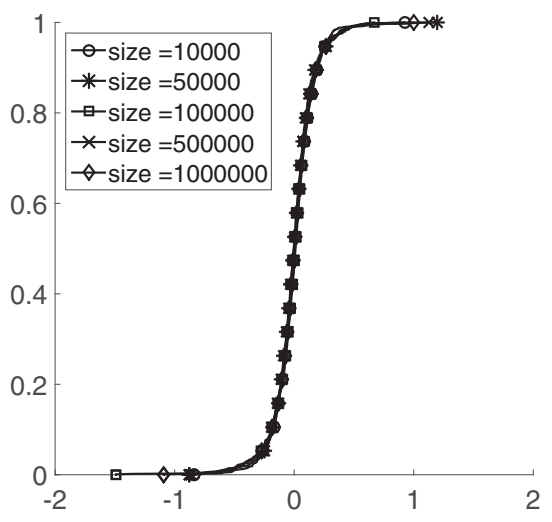

(a) I $-N^{1 / \gamma_{\min }-1}$

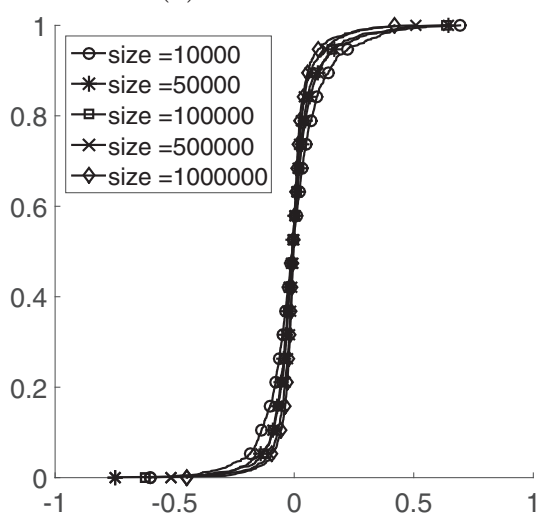

(d) II $-N^{\left(1 / \gamma_{\min }\right)-1}$

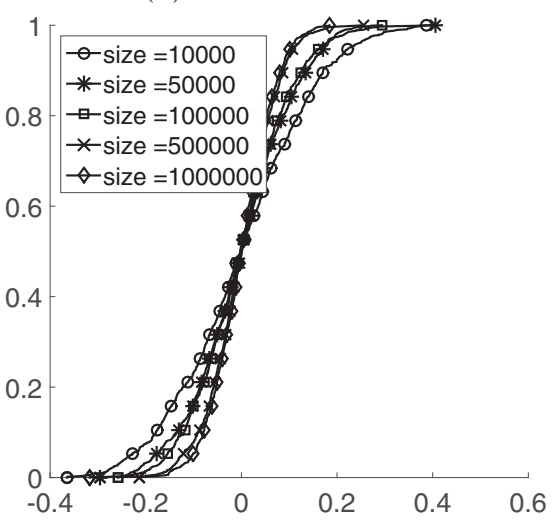

(g) III $-N^{\left(1 / \gamma_{\min }\right)-1}$

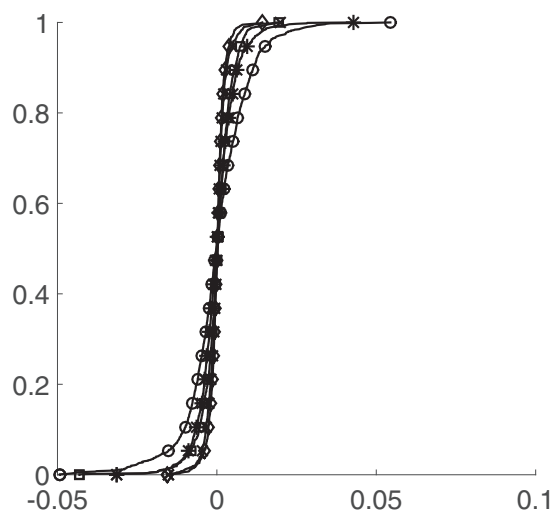

(b) I $-N^{\left(2 / \gamma_{+}\right)+\left(2 / \gamma_{-}\right)-3}$

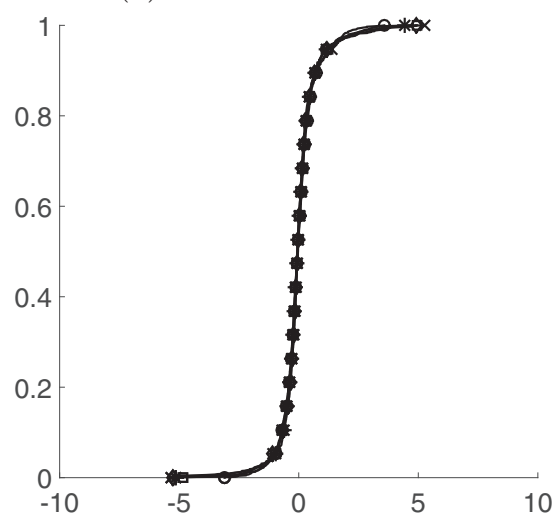

(e) II $-N^{\left(2 / \gamma_{+}\right)+\left(2 / \gamma_{-}\right)-3}$

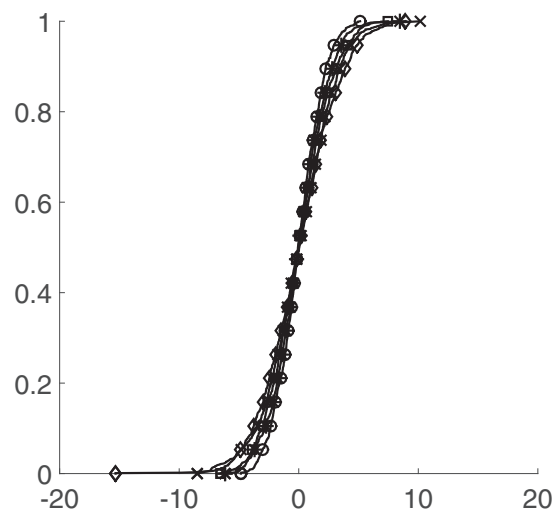

(h) III $-N^{\left(2 / \gamma_{+}\right)+\left(2 / \gamma_{-}\right)-3}$

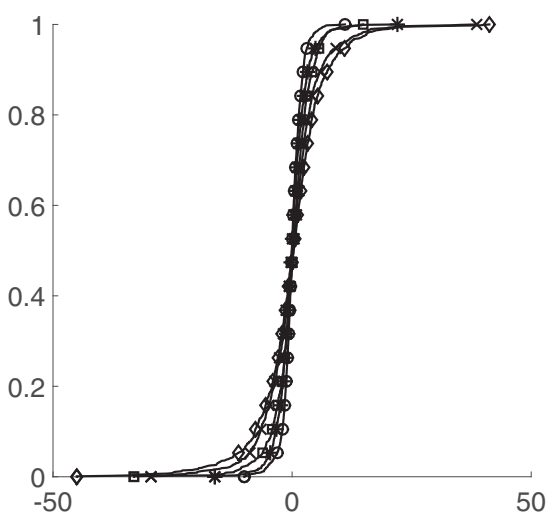

(c) I - $N^{-1 / 2}$

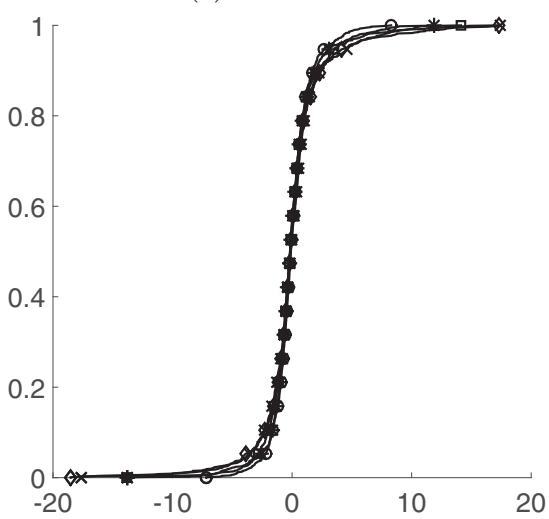

(f) II $-N^{-1 / 2}$

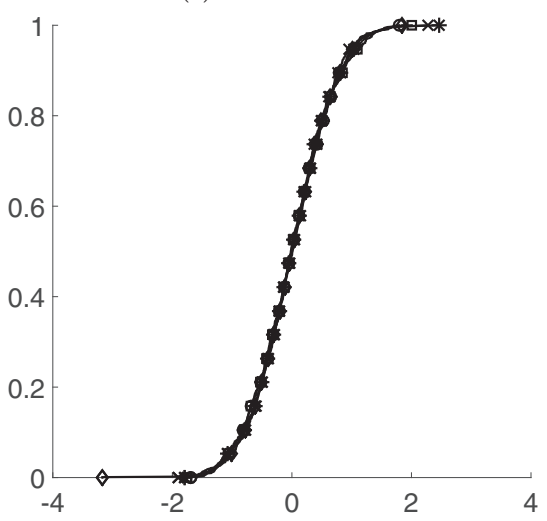

(i) III $-N^{-1 / 2}$

FIG. 8. Plots of the empirical cumulative distribution function of $\rho_{+}^{+}$for choices of $\gamma_{ \pm}$corresponding to points I, II, and III from Fig. 6, using the corresponding scaling.

converges to the distribution of two independent random variables as $N^{-1}$; see [21]. Because Spearman's rho and Kendall's tau on independent joint measurements are normal statistics [32], the scaling of their average is $N^{-1 / 2}$. Hence $\rho_{\alpha}^{\beta}$ for CM graphs scales as $N^{-1 / 2}$. Since an ECM graph is basically a CM graph where multiple edges are merged and self-loops are removed, it follows that the distributions for the degrees on both side of a randomly chosen edge differ from those of the CM by terms of the order $\sum_{i, j=1}^{N} E_{i j}^{c} / E$. Therefore, the scaling of $\rho_{+}^{-}$is determined by the largest term out of $N^{-1 / 2}$ and the scaling of $\sum_{i, j=1}^{N} E_{i j}^{c} / E$. Since the latter undergoes a phase transition, we actually have a three-stage phase transition for the scaling of $\rho_{+}^{-}$in the ECM. The first stage has scaling $N^{-1+1 / \gamma_{\min }}$ and holds for all $\gamma_{ \pm}$for which

$$
\frac{1}{\gamma_{\min }}-1 \leqslant \frac{2}{\gamma_{+}}+\frac{2}{\gamma_{-}}-3,
$$

since both correspond to upper bounds. The next region, $\gamma_{ \pm}$such that $2 / \gamma_{+}+2 / \gamma_{-}-3 \geqslant-1 / 2$, has scaling $N^{2 / \gamma_{+}+2 / \gamma_{-}-3}$. Outside this region we have normal scaling, $N^{-1 / 2}$. The different regions are displayed in Figure 6, while Table III shows the three scaling terms. We remark that the phase transitions of the scaling are smooth since they are induced by inequalities on the terms. 


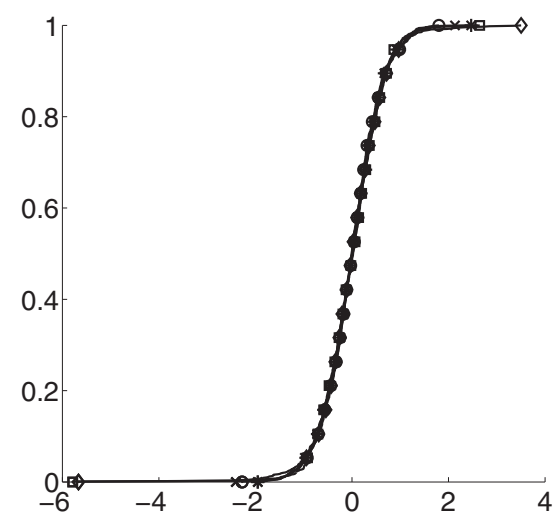

(a) I $-N^{-1 / 2}$

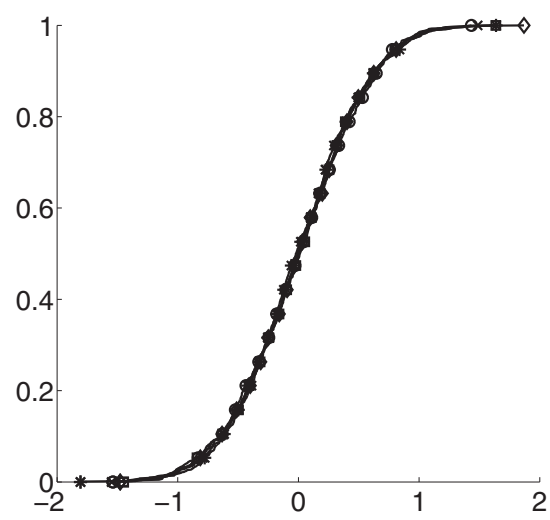

(b) II $-N^{-1 / 2}$

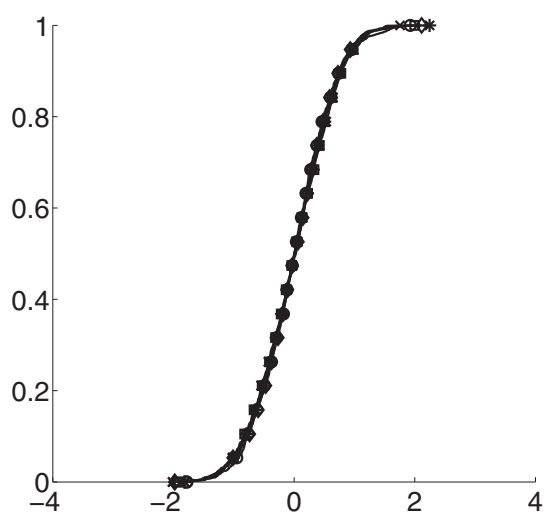

(c) III - $N^{-1 / 2}$

FIG. 9. Plots of the empirical cumulative distribution function of $\rho_{-}^{+}$for choices of $\gamma_{ \pm}$corresponding to points I, II, and III from Fig. 6, using square root scaling.

\section{Simulations}

In order to show the phase transitions we plotted the empirical cumulative distribution function (cdf) of $\rho_{+}^{-}$for the specific choices of $\gamma_{ \pm}$, corresponding to the points I, II, and III in Fig. 6. For each of the three points we shifted the empirical data by its average and multiplied it by $N^{-f\left(\gamma_{+}, \gamma_{-}\right)}$, for any of the three coefficients from Table III, corresponding to the different scaling areas $\mathrm{A}, \mathrm{B}$, and $\mathrm{C}$. The results are shown in Fig. 7. When the correct scaling is applied, the corresponding cdf plots should almost completely overlap and resemble the cdf of some limiting distribution. We observe that, for each of the three choices I, II, and III, this is the case when the corresponding scaling from its area, respectively $\mathrm{A}, \mathrm{B}$, and $\mathrm{C}$, is chosen.

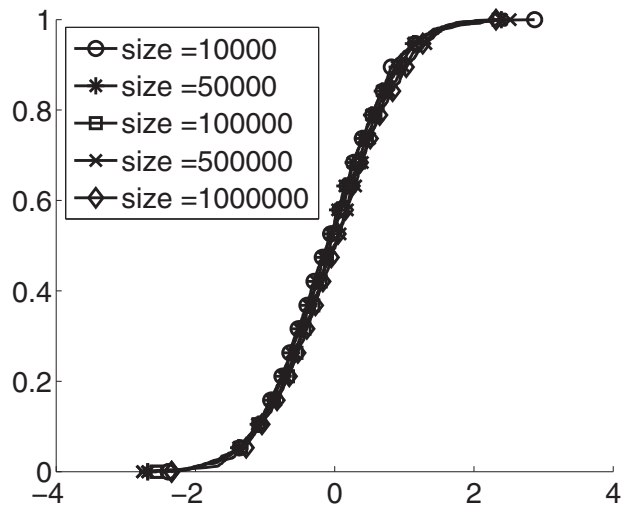

(a) Out-In

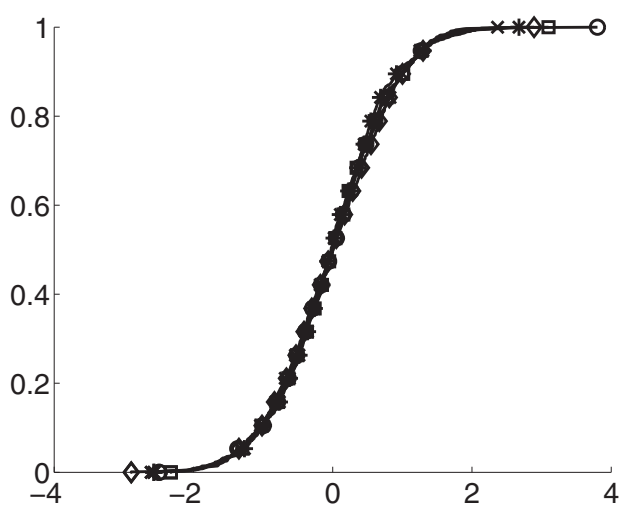

(c) Out-Out

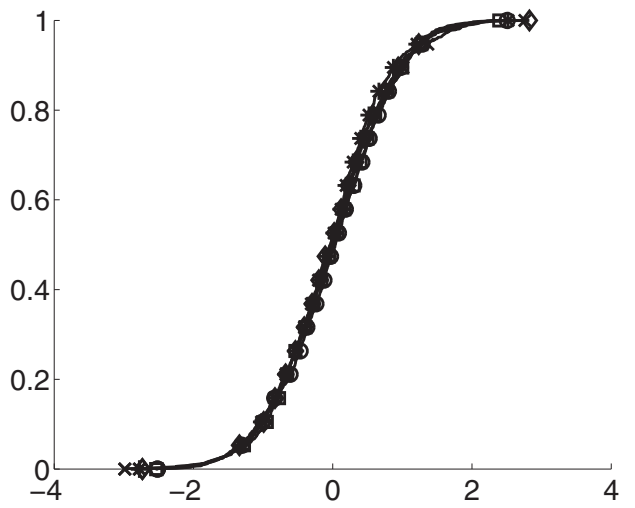

(b) In-Out

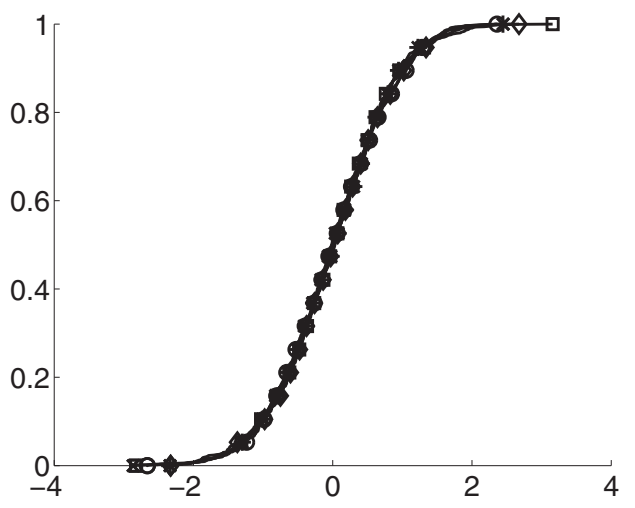

(d) In-In

FIG. 10. Plots of the empirical cumulative distribution of $\rho_{\alpha}^{\beta}$ for all four degree-degree dependency types for ECM graphs with $\gamma_{ \pm}=2.1$ of different sizes, scaled by $N^{-1 / 2}$. Each plot is based on $10^{3}$ realizations of the model. 


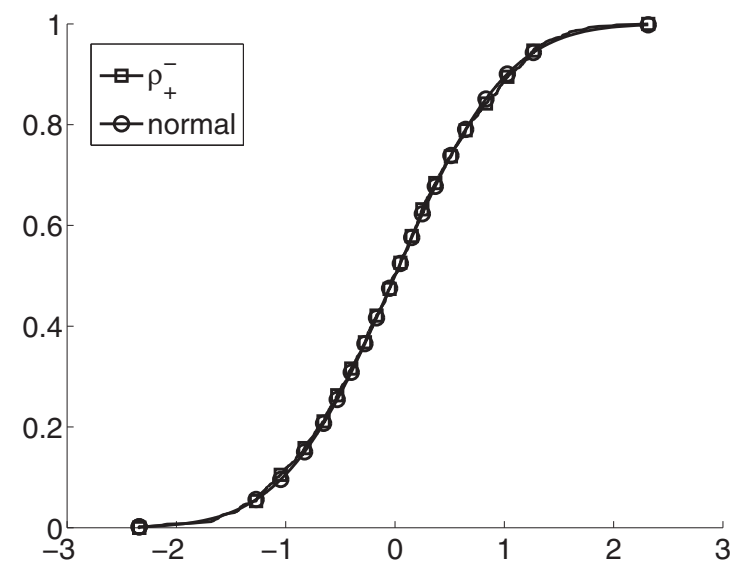

FIG. 11. Plot of the empirical cumulative distribution function of $\rho_{+}^{-}$for ECM graphs of size $10^{6}$ with $\gamma_{ \pm}=2.1$ and a normal cumulative distribution with $\mu=0$ and $\sigma^{2}=0.8$.

\section{SCALING OF DEGREE-DEGREE DEPENDENCIES FOR THE OTHER CASES}

In the previous section we completely characterized the scaling behavior of $\rho_{+}^{-}$for ECM graphs with infinite variance of the degrees. Here, we first discuss the remaining correlation types, $\rho_{+}^{+}, \rho_{-}^{-}$, and $\rho_{-}^{+}$in the infinite variance regime, and last we consider all four types in the finite variance regime.

The intuition behind the structural negative out-in dependencies was that multiple edges are more likely to exist between nodes of large out- and in-degrees. The other three types do not show negative correlations, see Figs. 5(b)-5(d), which we argued was due to the fact that the in- and out-degrees of a node in the ECM are independent. Nevertheless, the spread of both the out-out and in-in degree-degree dependency exhibits scaling with the same functions as the out-in dependency. This is illustrated in Fig. 8, where we plotted the empirical cumulative distribution of the out-out dependency for ECM graphs, for values of $\gamma_{ \pm}$corresponding to points I, II, and III from Fig. 6, scaled by the correct term for each of these points. This is because $\rho_{+}^{+}$again depends on the number of erased edges, through the out-degree of their source nodes. However, the out-degree of the target node of a removed edge can be both large or small, thus $\rho_{+}^{+}$in the ECM remains zero on average. By symmetry, the scaling for the in-in dependency is similar.

This nontrivial scaling is typical for the ECM. Recall that, in the $\mathrm{CM}, \rho_{\alpha}^{\beta}$ is a normal statistic and scales as $N^{-1 / 2}$ for any $\alpha, \beta$ because all degrees are independent random variables. This is exactly what we observe for the in-out degree-degree dependency, which, in contrast to the other three, is not biased towards removed edges. As we expect, here we have normal, square root scaling for ECM graphs for any choice of $\gamma_{ \pm}$. This can clearly be observed in Fig. 9, where we plotted the empirical cumulative distributions of $\rho_{-}^{+}$scaled by $N^{-1 / 2}$.

For the degree-degree dependencies in the finite variance regime we plotted the empirical cumulative distributions of $\rho_{\alpha}^{\beta}$, scaled by $N^{-1 / 2}$, in Fig. 10 . Since these are all completely similar, we took the plot for $\rho_{+}^{-}$for an ECM graph of size $10^{6}$ and compared it to a fitted normal distribution with $\mu=0$ and $\sigma^{2}=0.8$; see Fig. 11 . These plots strongly overlap, enforcing the claim that for ECM graphs with finite degree variance all four correlations are normal statistics.

\section{CONCLUSION AND DISCUSSION}

In this paper we analyzed degree-degree dependencies in the directed erased configuration model. We showed, Fig. 5, that in the infinite variance regime only the out-in dependency exhibits structural negative values, while all correlations behave similar when both degrees have finite variance, Fig. 4. We investigated the scaling of the structural negative out-in correlations. These undergo a phase transition in terms of the exponents $\gamma_{ \pm}$of the degree distributions (3), which we showed by establishing two upper bounds, (10) and (17), on the total average removed number of edges, both of which scale at different rates. Combining this with the square root scaling of Spearman's rho and Kendall's tau, we identified three regions, depending on $\gamma_{ \pm}$, with different scaling, Fig. 6, and illustrated their phase transitions in Fig. 7. Next, we considered the remaining three dependency types for the infinite variance regime. We showed, Fig. 8, that the scaling of the out-out and in-in correlations behaves similarly to the out-in, even though they do not exhibit structural negative values, while the in-out degree-degree dependency has square root scaling, Fig. 9. Finally we investigated the scaling for correlations when the degrees have finite variance. In this case all four types have square root scaling and the plots of the cumulative distributions are very similar, Fig. 10. This was confirmed when we compared the plot of $\rho_{+}^{-}$for ECM graphs of size $10^{6}$, with $\gamma_{ \pm}=2.1$, with that of a fitted normal distribution in Fig. 11 .

Our analysis shows that degree-degree dependencies in directed networks display nontrivial behavior in terms of scaling when the degrees have infinite variance. This scaling is important when doing statistical analysis of these measures or their impact on other processes on networks, for it determines their spread and hence enables to assess the significance of measurements.

We showed that degree-degree dependencies for degrees with finite variance, scaled by $N^{-1 / 2}$, converge to a normal distribution with zero mean. We have not yet been able to determine the variance of these distributions as a function of the tail exponents $\gamma_{ \pm}$which would completely characterize their behavior.

For three of the four correlation types in the infinite variance regime, we did not determine the limiting distributions. This is mainly due to the fact that we expect these to be stable distributions, since one of the three scaling regions is due to the central limit theorem for regularly varying random variables, whose limits are stable distributions. Although these distributions have a well defined characteristic function, their density function, in general, does not have an analytical expression. Moreover, we are dealing with discrete data and simulation of such distributions is a field of its own. Nevertheless, we do expect that central limit theorems for degree-degree dependencies can be formulated and proven, which would fully complete their statistical analysis.

Finally, our empirical results clearly show the analytically derived phase transitions. However, the region with the $N^{\left(2 / \gamma_{+}\right)+\left(2 / \gamma_{-}\right)-3}$ scaling is less distinct than the other two. One of the possible reasons for this is that within the area where 
this scaling applies, the difference in value with the other two terms is small. We therefore picked point II in Fig. 6 such that this difference was large enough to distinctly show this scaling visually in the plots.

We close by strongly suggesting to use the ECM as a null model for analysis of degree-degree dependencies, both for determining their impact on processes as well as significance. Although, for the latter, values are often compared to averages using the rewiring model [5], we emphasize that fixing the degrees imposes strong constraints on the possible simple graphs that can be generated. Moreover, in real-life networks, not only wiring but also the degrees of the nodes are a result of a random process. Therefore, in a null model, it seems more natural to fix only general properties of the network, such as degree distributions.

\section{ACKNOWLEDGMENTS}

All computations in this paper where done using the FASTUTIL package and the WEBGRAPH framework [33], from the Laboratory for Web Algorithmics [34]. This work is supported by the EU-FET Open grant NADINE (288956).
[1] A. Vázquez and Y. Moreno, Phys. Rev. E 67, 015101 (2003).

[2] A. Srivastava, B. Mitra, F. Peruani, and N. Ganguly, in IEEE Conference on Computer Communications Workshops (INFOCOM WKSHPS), 2011 (IEEE, Piscataway, NJ, 2011), pp. 1076-1081.

[3] M. Boguná and R. Pastor-Satorras, Phys. Rev. E 66, 047104 (2002).

[4] M. Boguná, R. Pastor-Satorras, and A. Vespignani, Phys. Rev. Lett. 90, 028701 (2003).

[5] S. Maslov and K. Sneppen, Science 296, 910 (2002).

[6] B. Bollobás, Eur. J. Combinator. 1, 311 (1980).

[7] M. Molloy and B. Reed, Random Struct. Algorithms 6, 161 (1995).

[8] M. E. Newman, S. H. Strogatz, and D. J. Watts, Phys. Rev. E 64, 026118 (2001).

[9] J. Blitzstein and P. Diaconis, Int. Math. 6, 489 (2011).

[10] C. I. Del Genio, H. Kim, Z. Toroczkai, and K. E. Bassler, PloS one 5, e10012 (2010).

[11] H. Kim, C. I. Del Genio, K. E. Bassler, and Z. Toroczkai, New J. Phys. 14, 023012 (2012).

[12] T. Squartini and D. Garlaschelli, New J. Phys. 13, 083001 (2011).

[13] M. Catanzaro, M. Boguñá, and R. Pastor-Satorras, Phys. Rev. E 71, 027103 (2005).

[14] S. Maslov, K. Sneppen, and A. Zaliznyak, Physica A 333, 529 (2004).

[15] J. Park and M. E. J. Newman, Phys. Rev. E 68, 026112 (2003).

[16] S. N. Dorogovtsev and J. F. Mendes, Adv. Phys. 51, 1079 (2002).
[17] M. Boguná, R. Pastor-Satorras, and A. Vespignani, Eur. Phys. J. B 38, 205 (2004).

[18] A. Clauset, C. R. Shalizi, and M. E. Newman, SIAM Rev. 51, 661 (2009).

[19] P. Bloem, http://github.com/Data2Semantics/powerlaws.

[20] N. Chen and M. Olvera-Cravioto, Stoch. Syst. 3, 147 (2013).

[21] P. van der Hoorn and N. Litvak, Moscow J. Combinator. Number Theory 4, 45 (2014)

[22] J. G. Foster, D. V. Foster, P. Grassberger, and M. Paczuski, Proc. Natl. Acad. Sci. USA 107, 10815 (2010).

[23] P. van der Hoorn and N. Litvak, Internet Math. 11, 155 (2015).

[24] M. E. J. Newman, Phys. Rev. Lett. 89, 208701 (2002).

[25] N. Litvak and R. van der Hofstad, Phys. Rev. E 87, 022801 (2013).

[26] S. N. Dorogovtsev, A. L. Ferreira, A. V. Goltsev, and J. F. F. Mendes, Phys. Rev. E 81, 031135 (2010).

[27] C. Spearman, Am. J. Psychol. 15, 72 (1904).

[28] M. G. Kendall, Biometrika 30, 81 (1938).

[29] R. van der Hofstad, G. Hooghiemstra, and P. Van Mieghem, Random Struct. Algorithms 27, 76 (2005).

[30] N. H. Bingham and R. A. Doney, Adv. Appl. Probab. 6, 711 (1974).

[31] W. Whitt, Stochastic-Process Limits: An Introduction to Stochastic-process Limits and Their Application to Queues (Springer, Berlin, 2002).

[32] W. Hoeffding, Ann. Math. Stat. 19, 293 (1948).

[33] P. Boldi and S. Vigna, in Proceedings of the 13th International Conference on the World Wide Web (ACM, New York, 2004), pp. 595-602.

[34] http://law.di.unimi.it/software.php. 